\title{
EFEITO DA ADIÇÃO DE INGREDIENTES NA COR DE POLPA DE GOIABA
}

\author{
RENATA TORREZAN * \\ VERA LÚCIA PUPO FERREIRA * * \\ KATUMI YOTSUYANAGI *** \\ JOSÉ GILBERTO JARDINE **** \\ ALFREDO DE ALMEIDA VITALI ***
}

\begin{abstract}
Sete amostras de polpa de goiaba estáveis ao armazenamento a $25 \stackrel{\circ}{\circ} \mathrm{C}$, preservadas com o uso da tecnologia de métodos combinados e tratadas com sacarose, ácido cítrico e sorbato de potássio foram avaliadas quanto às variações de cor em relação à amostra original (sem tratamento). As medidas de cor foram realizadas por reflexão em espectrofotômetro. Com os dados obtidos calculou-se o croma e a diferença total da cor em relação à amostra original. Todos os tratamentos foram avaliados visualmente e ordenados quanto à intensidade de luminosidade, vermelho $e$ amarelo por uma equipe de julgadores com discriminação superior para cor. Todos as amostras apresentaram diminuição da luminosidade, do teor de vermelho e de amarelo em relação à polpa de goiaba inicial, com exceção do tratamento 1 ( $0 \%$ de sacarose, $0,75 \%$ de ácido cítrico e $0,10 \%$ de sorbato de potássio), que obteve dados de cor bastante próximos aos da polpa original.
\end{abstract}

\section{INTRODUÇÃO}

O mundo é percebido pelo ser humano mediante seus sentidos, dos quais a visão é o mais marcante. O impacto visual não envolve apenas a forma e aspecto dos objetos, mas também a cor. Dos três principais atributos de qualidade dos alimentos - cor, sabor e textura - a cor torna-se cada vez mais importante, à medida que os alimentos são expostos e vendidos (4).

* $\quad$ M.Sc. em Tecnologia de Alimentos, Embrapa Agroindústria de Alimentos, Rio de Janeiro - RJ. (e-mail: torrezan@ctaa.embrapa.br).

** D.Sc. em Tecnologia de Alimentos, Consultora, Campinas - SP.

*** D.Sc., Pesquisadores, Instituto de Tecnologia de Alimentos (ITAL), Campinas - SP.

**** D.Sc. em Tecnologia de Alimentos, Embrapa Informática Agropecuária, Campinas SP. (e-mail: jardine@cnptia.embrapa.br). 
$\mathrm{Na}$ indústria de alimentos, a cor é importante parâmetro empregado no controle de qualidade. É pela cor do extrato de tomate e do suco de laranja, por exemplo, que estes alcançam as melhores classificações e efetivamente maiores preços. Na torração do café, do cacau e do amendoim, a cor do grão define exatamente quando está terminado o processo. Muitas vezes, a cor é adicionada aos alimentos para conferir ou aumentar sua apetitosidade. A verdade é que se aceita ou não determinado alimento, em primeiro lugar, com os olhos, ou seja, pela cor. Se a cor não for atraente, apesar da aparência (aspecto e forma) e o odor o serem, dificilmente o alimento será ingerido ou pelo menos provado (4).

A cor das polpas de frutas está relacionada com sua qualidade e também com o tipo e quantidade de pigmentos presentes. No caso da polpa de goiaba, a cor varia do amarelo ao vermelho como resultado da presença de carotenóides. Os principais carotenóides presentes são o $\beta$-caroteno e licopeno $(14,17)$.

Tanto nos processos industriais, quanto nos comerciais, ou puramente de ordem emocional são utilizados os olhos na escolha de produtos competitivos, motivo pelo qual sofisticados métodos objetivos de medição da cor têm sido desenvolvidos (4). A cor de polpa de frutas pode ser medida por espectrofotometria de transmissão ou por reflexão, desde que se propicie a reflexão da luz transmitida através da polpa por meio de técnicas adequadas (7).

A preservação dos alimentos pelo uso dos métodos combinados resumese na aplicação de parâmetros combinados (ou obstáculos), que podem agir sinergisticamente para inibir ou retardar o crescimento microbiano, resultando em produtos estáveis em temperatura ambiente $(6,2)$. Cada alimento seguro e estável requer certo tipo de obstáculos, que diferem em qualidade e intensidade de acordo com cada produto em particular, de modo a manter a população natural de microrganismos sob controle (11).

Muitos trabalhos usando métodos combinados têm sido realizados nos mais diversos países do mundo, utilizando os mais variados produtos. Encontra-se razoável quantidade de trabalhos efetuados sobre frutas, principalmente em países americanos (3) e na Índia (10). Alguns destes trabalhos afirmam que o uso dos métodos combinados preserva as características sensoriais (cor, textura e sabor) dos produtos desenvolvidos $(1,9,12)$.

JAYARAMAN et al (9) empreenderam estudos de preparação de goiaba fatiada de umidade intermediária. Este produto foi embalado em latas e estocado a $0{ }^{\circ} \mathrm{C}$, temperatura ambiente $\left(27-30{ }^{\circ} \mathrm{C}\right)$ e a $37{ }^{\circ} \mathrm{C}$, sendo os parâmetros cor, textura e sabor avaliados periodicamente. escurecimento das amostras foi medido com colorímetro Lovibond e a cor expressa em vermelho, amarelo e azul. Os resultados obtidos revelaram 
que as amostras não sofreram variação na cor, textura e sabor quando estocadas a $0 \stackrel{\circ}{\circ}$, durante 06 meses de armazenamento. As amostras mantidas em temperatura ambiente revelaram-se aceitáveis até 06 meses, apesar de apresentarem ligeiro escurecimento. Já as estocadas a $37{ }^{\circ} \mathrm{C}$ mostraram-se aceitáveis até 03 meses, pois sofreram intenso escurecimento e alteração no sabor.

ALZAMORA et al. (1) trabalharam com preservação de abacaxis por métodos combinados em temperatura ambiente $\left(27 \pm 1^{\circ} \mathrm{O}\right)$, durante quatro meses de estocagem. Os obstáculos usados foram: tratamento térmico brando, redução de atividade de água e pH e adição de bissulfito de sódio e sorbato de potássio. $\mathrm{Na}$ avaliação de cor foi observada diminuição da luminosidade e intensidade de verde e de amarelo ao longo do período de estocagem. As mudanças observadas na cor do produto não comprometeram a sua aceitabilidade, porém revelaram que 0 processamento e a estocagem favoreceram o aumento do escurecimento com ligeira descoloração das fatias.

LÓPEZ-MALO et al. (12) utilizaram métodos combinados na preservação de mamão fatiado, minimamente processado e estocado a 5 e $25 \stackrel{\circ}{\circ}$, por cinco meses. A avaliação da cor, textura e sabor revelaram que não houve diferença significativa entre as amostras em função da temperatura de estocagem. Foi observado que o croma permaneceu praticamente constante em ambas as temperaturas de estocagem. Entretanto, a luminosidade decresceu de $10-20 \%$ durante a estocagem, provavelmente devido ao escurecimento não enzimático, decorrente do alto conteúdo de açúcares redutores. As mudanças de cor não afetaram significativamente a aceitabilidade sensorial do produto.

O objetivo deste trabalho foi avaliar objetiva e sensorialmente as variações de cor em amostras de polpa de goiaba, tratadas com diferentes concentrações de sacarose, ácido cítrico e sorbato de potássio, estáveis ao armazenamento a $25{ }^{\circ} \mathrm{C}$, em relação a cor da amostra de polpa de goiaba original (sem tratamento).

\section{MATERIAL E MÉTODOS}

\subsection{MATERIAL}

\subsubsection{Matéria-prima}

- Polpa de goiaba (variedade IAC-4), concentrada (15 $5^{\circ}$ Brix) pelo processo "hot-fill" e processada em Monte Alto/SP, safra de 1995 (sem tratamento); 
- amostras de polpa de goiaba (15을 Brix), estáveis ao armazenamento a $25 \stackrel{\circ}{ } \mathrm{C}$, inoculadas com $10^{3} \mathrm{UFC} / \mathrm{g}$ de microrganismos, tratadas com sacarose, ácido cítrico e sorbato de potássio, nas condições mencionadas na Tabela 1.

\section{TABELA 1 - CONDIÇÕES DOS TRATAMENTOS DAS AMOSTRAS DE POLPA DE GOIABA ESTÁVEIS AO ARMAZENAMENTO A $25 \stackrel{\circ}{\circ}$}

\begin{tabular}{lccc}
\hline & $\begin{array}{c}\text { Sacarose } \\
(\%)\end{array}$ & $\begin{array}{c}\text { Ácido cítrico } \\
(\%)\end{array}$ & $\begin{array}{c}\text { Sorbato de potássio } \\
(\%)\end{array}$ \\
\hline 150 dias de & & & \\
armazenamento & & 0,75 & 0,10 \\
Tratamento 1 & 0 & 0,75 & 0,06 \\
Tratamento 2 & 54 & 0,75 & 0,10 \\
Tratamento 3 & 50 & & \\
120 dias de & & & \\
armazenamento & & 0,22 & 0,06 \\
Tratamento 4 & 46,09 & 0,75 & 0,06 \\
Tratamento 5 & 54 & - & 0,06 \\
Tratamento 6 & 54 & 0,75 & 0,05 \\
Tratamento 7 & 54 & & \\
\hline
\end{tabular}

\subsubsection{Outros materiais}

Soluto - sacarose "Cristalçucar" (Copersucar LTDA).

Ácido cítrico p.a. (monohidratado) Merck.

Conservador - sorbato de potássio USP (99,5\% de pureza), Merse Ltda. Inóculo $-10^{3} \mathrm{UFC} / \mathrm{g}$ formado por bolores, leveduras e bactérias lácticas.

\subsection{MÉTODO}

\subsubsection{Preparo da amostra}

Todos os tratamentos foram definidos de acordo com o delineamento experimental adotado por TORREZAN (16) e preparados no mesmo dia. Cada formulação continha $2.500 \mathrm{~g}$ ( 3 vidros, com tampas rosqueáveis de $600 \mathrm{~g}$ e o restante utilizado em outras análises iniciais). A seqüência das adições nas formulações foi polpa, ácido, açúcar, conservador e inóculo, com tempo de mistura de cerca de 5 minutos, em batedeira planetária (Kitchen Aid, modelo K5-A, The Hobart MFG Co., EUA). Os frascos foram mantidos a $25{ }^{\circ} \mathrm{C}$ e acondicionados em caixas de papelão fechadas. Os tratamentos que permaneceram microbiológica e quimicamente estávéis ao armazenamento a $25^{\circ} \mathrm{C}$ foram analisados quanto às variações de cor em relação a amostra original (sem tratamento). 


\subsubsection{Análise de cor}

As sete amostras tratadas e uma amostra da polpa original (sem tratamento) foram passadas em desaerador de laboratório a vácuo (Cunnigton and Cooper Ltda) até a ausência de bolhas de ar. A seguir foram colocadas em cápsula de fundo de quartzo e as leituras efetuadas na faixa de 370 a $740 \mathrm{~nm}$, tendo as amostras opacidade comprovada para a medição da cor.

As medidas de cor foram feitas por reflexão em espectrofotômetro Color Eye COMCOR 1500 Plus (COMEXIM, São Paulo), usando iluminante C, ângulo de observação de $2^{\circ}$, sistema $L^{*} a^{*} b^{*}$ CIE ("Commission Internationale de L' Eclairage"), abertura normal e configuração DREOL, sendo que $L^{*}$ mede a luminosidade, isto é, o quanto a cor é clara ou escura, $a^{*}$ mede a intensidade de vermelho e $b^{*}$ mede a intensidade de amarelo. A metodologia adotada foi proposta por FERREIRA (4).

Com os dados obtidos (médias de duas determinações) calculou-se o croma $\left(\mathrm{C}=\left[(\mathrm{a})^{2}+(\mathrm{b})^{2}\right]^{1 / 2}\right)$ e a diferença total da cor em relação à amostra original $\left(\Delta E=\left[\left(L_{p}-L_{a}\right)^{2}+\left(a_{p}-a_{a}\right)^{2}+\left(b_{p}-b_{a}\right)^{2}\right)\right]^{1 /}$. Os índices $\underline{p}$ e a referemse, respectivamente, a amostra original da polpa e as demais amostras testadas (7). Croma é a "força" da cor, é a qualidade pela qual se distingue a cor fraca da forte, é a intensidade de um tom distinto ou a intensidade de cor. As diferenças totais de cor são importantes na indústria para estipular suas variações aceitáveis, ou seja, as tolerâncias (4).

As mesmas amostras foram também avaliadas visualmente e ordenadas quanto à intensidade de luminosidade, vermelho e amarelo por equipe de 9 julgadores com discriminação superior para cor (5). As amostras foram colocadas em xícaras brancas codificadas com números de 3 dígitos, niveladas e apresentadas segundo o delineamento de blocos completos aleatorizados aos julgadores, em cabine de luz Super Skylight (Macbeth, EUA), sob o iluminante C. Os dados da avaliação visual foram analisados pelo Teste de Friedman, utilizando-se a Tabela de Newell e MacFarlane (13). Os coeficientes de correlação foram calculados segundo HOFFMANN \& VIEIRA (8) e SPIEGEL (15).

\section{RESULTADOS E DISCUSSÃo}

Durante a realização dos experimentos com polpas de goiaba foi observado, de maneira geral, que estas polpas ao serem tratadas com açúcar, logo após a mistura, aparentemente ficavam mais vermelhas e "mais brilhantes". Este fato foi verificado até a adição de cerca de $50 \%$ de sacarose $(\% \mathrm{p} / \mathrm{p})$. Porém, quando o nível de adição era muito elevado, 
acima de $60 \%$ de sacarose ( $\% \mathrm{p} / \mathrm{p})$, as polpas tornavam-se opacas e de tom mais alaranjado. Assim, efetuou-se avaliação visual da cor, em termos físicos e pela ordenação visual, apenas das amostras dos tratamentos que se mostraram estáveis ao armazenamento de, no mínimo, 120 dias a $25{ }^{\circ} \mathrm{C}$ (Tabela 1), em comparação à amostra inicial da polpa de goiaba (sem tratamento).

A Figura 1 apresenta os dados da avaliação física da cor dos tratamentos de polpa de goiaba, segundo os valores médios de $L^{*}, a^{*}, b^{*}(C I E)$ e os calculados de $\Delta E$ e $C$. Observou-se que em termos de luminosidade $\left(L^{*}\right)$, a amostra sem tratamento e a do tratamento 1 obtiveram os maiores valores. O tratamento 6 revelou os menores valores enquanto os demais tratamentos apresentaram valores intermediários. De maneira geral, nos tratamentos aplicados houve diminuição da luminosidade, chegando a cerca de $60 \%$ de redução para o tratamento 6 . Tal redução foi superior a observada por LÓPEZ-MALO et al. (12), provavelmente devido ao fato destes tratamentos apresentarem nível de adição de açúcar muito superior, favorecendo a ocorrência de escurecimento não enzimático. Os teores de vermelho $\left(\mathrm{a}^{*}\right)$ foram maiores na amostra sem tratamento e nos tratamentos 1 e 3 e menores para os tratamentos 5, 6 e 2. Os tratamentos diminuíram os teores de vermelho das amostras, exceto nos tratamentos 1 e 3, ocorrendo a variação máxima (cerca de 11\%), para o tratamento 6 . Os teores de amarelo $\left(b^{*}\right)$ diminuíram com os tratamentos, atingindo cerca de $20 \%$ de variação para o tratamento 6 , exceto para o tratamento 1 que apresentou valor próximo ao da amostra sem tratamento. O croma também diminuiu com os tratamentos, variação de até cerca de $16 \%$, sendo 0 tratamento 1 bastante semelhante a polpa inicial (sem tratamento).

Para a diferença total de cor $(\Delta e)$, em relação a amostra sem tratamento, o tratamento 1 foi o que apresentou o menor valor. Os demais apresentaram valores maiores de $\Delta \mathrm{e}$, distanciando-se mais em termos de cor da amostra inicial. $O$ tratamento 6 foi o que apresentou maior diferença, caracterizada pelos menores valores de $\mathrm{I}^{*}, \mathrm{a}^{*} \mathrm{e} \mathrm{b}^{*}$ obtidos. Os valores de croma (c), excetuando-se o tratamento 1 , diminuíram com os tratamentos, sendo 0 menor valor obtido pelo tratamento 6 .

O resultado da avaliação sensorial (ordenação) das polpas de goiaba consta das Tabelas 2, 3 e 4. Quanto à ordenação pela luminosidade (Tabela 2), o tratamento 6 foi considerado pelos julgadores como o mais escuro e a polpa inicial (sem tratamento) como a mais clara. Pela aplicação do Teste de Friedman, a polpa inicial diferiu quanto à luminosidade ao nível de $1 \%$ de probabilidade dos tratamentos 4,5 e 6 , e do tratamento 7 ao nível de $5 \%$ de probabilidade. O tratamento 1 diferiu significativamente ao nível de $1 \%$ dos tratamentos 4,5 e 6 . O tratamento 2 diferiu ao nível de $5 \%$ do tratamento 6 e o tratamento 3 diferiu ao nível de $5 \%$ dos tratamentos 5 e 6 . A ordenação média das polpas (mais claro para mais escuro) foi: polpa inicial e tratamentos $1,3,2,7,4,5$ e 6 . 
FIGURA 1 - DADOS DE COR NO SISTEMA L*a*b* CIE DE POLPAS DE GOIABA ESTÁVEIS, SENDO (a) LUMINOSIDADE - L; (b) TEOR DE VERMELHO - a; (c) TEOR DE AMARELO - b; (d) DIFERENÇA TOTAL DE COR - $\triangle E$; (e) CROMA - C

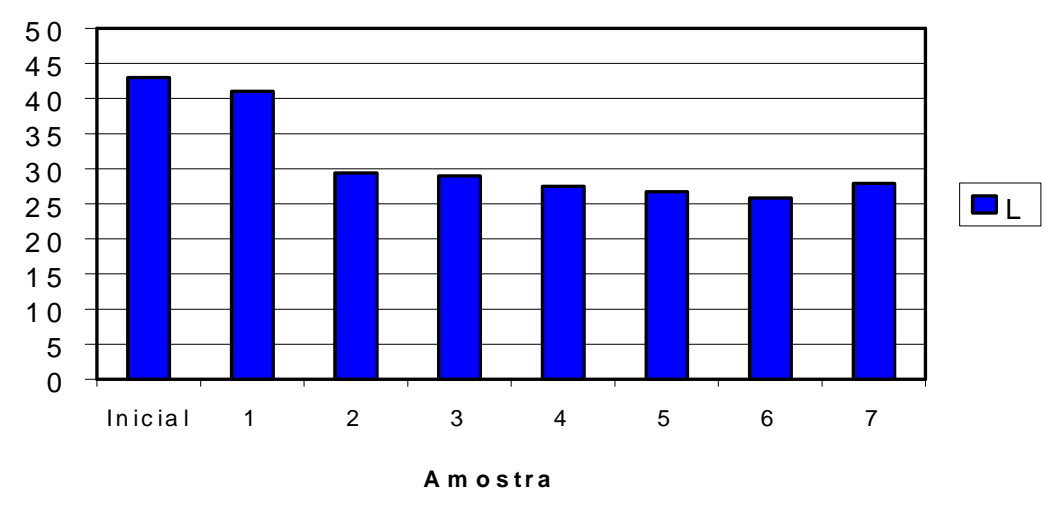

(a)

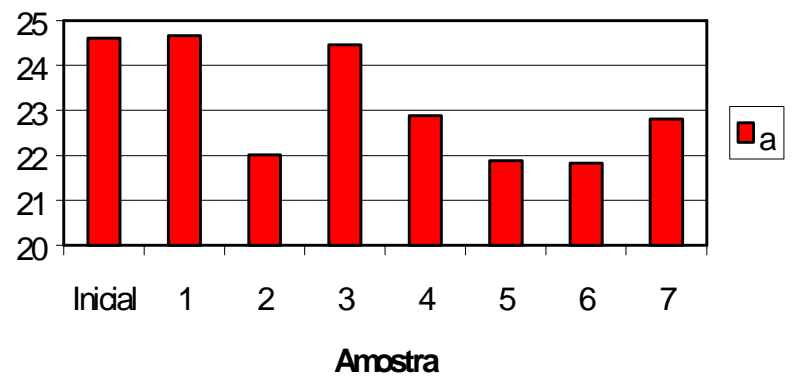

(b)

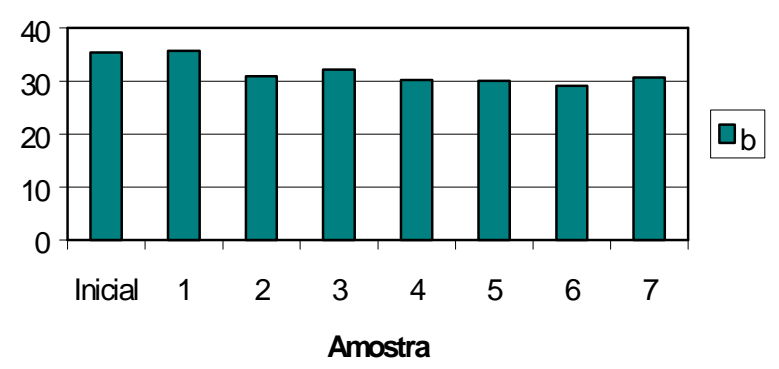

(c)

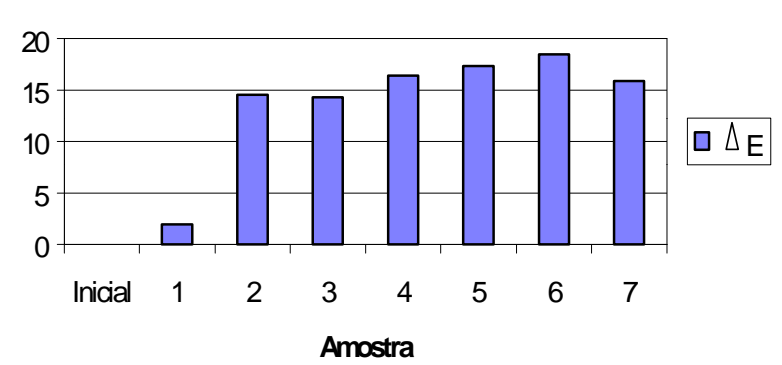

(d)

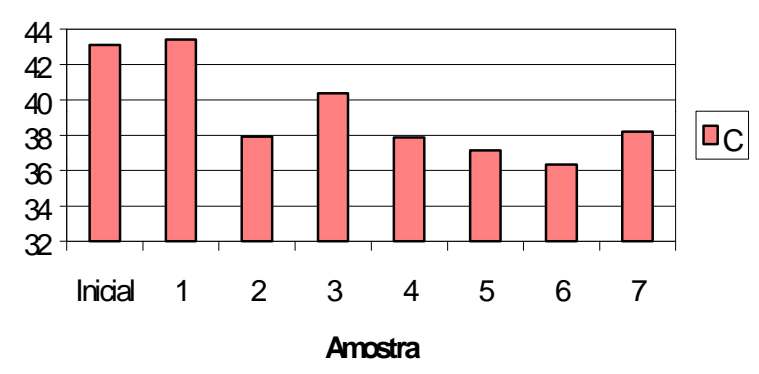

(e) 
A ordenação visual das polpas, quanto ao teor de vermelho (Tabela 3), mostrou que a polpa inicial diferiu ao nível de $1 \%$ de probabilidade dos tratamentos 4,5 e 6 e ao nível de $5 \%$ do tratamento 7 . O tratamento 1 diferiu ao nível de $1 \%$ dos tratamentos 4, 5 e 6 . Em termos de ordenação das polpas (mais vermelho para menos vermelho) foram obtidos os seguintes resultados: $6,4,5,7,3,2,1$ e polpa inicial. Pode-se observar que a polpa inicial, ou seja, sem nenhum tratamento foi considerada a menos vermelha seguida pelo tratamento 1 .

TABELA 2 - SOMA DAS ORDENACCÕES VISUAIS DA LUMINOSIDADE COM A APLICAÇÃO DO TESTE DE FRIEDMAN (TABELA DE NEWELL E MACFARLANE) DAS POLPAS DE GOIABA ESTÁVEIS

\begin{tabular}{ccccccccc}
\hline T RATAMENTO & $\begin{array}{c}\text { 'olpa } \\
\text { jicial }\end{array}$ & 1 & 2 & 3 & 4 & 5 & 6 & 7 \\
\hline Soma das ordens & 72 & 63 & 45 & 54 & 24 & 20 & 10 & 36 \\
\hline 1 & & 9 & 27 & 18 & $48^{\mathrm{a}}$ & $52^{\mathrm{a}}$ & $62^{\mathrm{a}}$ & $36^{\mathrm{a}}$ \\
2 & & & 18 & 9 & $39^{\mathrm{b}}$ & $43^{\mathrm{b}}$ & $53^{\mathrm{b}}$ & 27 \\
3 & & & & 9 & 21 & 25 & $35^{\mathrm{c}}$ & 9 \\
4 & & & & & 30 & $34^{\mathrm{d}}$ & $44^{\mathrm{d}}$ & 18 \\
5 & & & & & & 4 & 14 & 12 \\
6 & & & & & & & 10 & 16 \\
7 & & & & & & & & 26 \\
\hline
\end{tabular}

$a=$ significativamente diferente da polpa inicial $(p \leq 5) ; b=$ significativamente diferente do tratamento $1(p=1) ; c=$ significativamente diferente do tratamento $2(p=5) ; d=$ significativamente diferente do tratamento $3(p=5)$.

Obs.: Quanto menores as somas das ordens, mais escuras são as amostras.

TABELA 3 - SOMA DAS ORDENAÇÕES VISUAIS DO VERMELHO COM A APLICAÇÃO DO TESTE DE FRIEDMAN (TABELA DE NEWELL E MACFARLANE) DAS POLPAS DE GOIABA ESTÁVEIS

\begin{tabular}{ccccccccc}
\hline 7 RATAMENTO & $\begin{array}{c}\text { 'olpa } \\
\text { i licial }\end{array}$ & 1 & 2 & 3 & 4 & 5 & 6 & 7 \\
\hline Soma das ordens & 71 & 64 & 43 & 42 & 25 & 28 & 14 & 37 \\
\hline 1 & & 7 & 28 & 29 & $46^{\mathrm{a}}$ & $43^{\mathrm{a}}$ & $57^{\mathrm{a}}$ & $34^{\mathrm{a}}$ \\
2 & & & 21 & 22 & $39^{\mathrm{b}}$ & $36^{\mathrm{b}}$ & $50^{\mathrm{b}}$ & 27 \\
3 & & & & 1 & 18 & 15 & 29 & 6 \\
4 & & & & & 17 & 14 & 28 & 5 \\
5 & & & & & & 3 & 11 & 12 \\
6 & & & & & & & 14 & 9 \\
7 & & & & & & & & 23 \\
\hline
\end{tabular}

$a=$ significativamente diferente da polpa inicial $(p \leq 5) ; b=$ significativamente diferente do tratamento $1(p=1)$.

Obs.: Quanto menores as somas das ordens, mais vermelhas são as amostras. 
Quanto ao teor de amarelo (Tabela 4) observou-se que a polpa inicial diferiu significativamente ao nível de $1 \%$ de probabilidade, dos tratamentos 4,5 e 6 e ao nível de $5 \%$ de probabilidade do tratamento 7 . O tratamento 1 diferiu, estatisticamente ao nível de $1 \%$, dos tratamentos 4,5 e 6 , e os tratamentos 2 e 3 diferiram, significativamente ao nível de $1 \%$, do tratamento 6 . A ordenação das polpas (mais amarelo para menos amarelo) resultou em: polpa inicial, 1, 3, 2, 7, 4, 5 e 6.

\section{TABELA 4 - SOMA DAS ORDENACCÕES VISUAIS DO AMARELO COM A APLICAÇÃO DO TESTE DE FRIEDMAN (TABELA DE NEWELL E MACFARLANE) DAS POLPAS DE GOIABA ESTÁVEIS}

\begin{tabular}{ccccccccc}
\hline 7 RATAMENTO & $\begin{array}{c}\text { 'olpa } \\
\text { incial }\end{array}$ & 1 & 2 & 3 & 4 & 5 & 6 & 7 \\
\hline Soma das ordens & 9 & 18 & 33 & 32 & 56 & 61 & 70 & 45 \\
\hline 1 & & 9 & 24 & 23 & $47^{\mathrm{a}}$ & $52^{\mathrm{a}}$ & $61^{\mathrm{a}}$ & $36^{\mathrm{a}}$ \\
2 & & & 15 & 14 & $38^{\mathrm{b}}$ & $43^{\mathrm{b}}$ & $52^{\mathrm{b}}$ & 27 \\
3 & & & & 1 & 23 & 28 & $37^{\mathrm{c}}$ & 12 \\
4 & & & & & 24 & 29 & $38^{\mathrm{d}}$ & 13 \\
5 & & & & & & 5 & 14 & 11 \\
6 & & & & & & & 9 & 16 \\
7 & & & & & & & & 25 \\
\hline
\end{tabular}

$a=$ significativamente diferente da polpa inicial $(p \leq 5) ; b=$ significativamente diferente do tratamento $1(p=1) ; c=$ significativamente diferente do tratamento $2(p=1) ; d=$ significativamente diferente do tratamento $3(p=1)$.

Obs.: Quanto menores as somas das ordens, mais amarelas são as amostras.

De forma geral, $L^{*}$ diminuiu com o aumento do teor de sacarose, $a^{*}$ foi maior para os teores máximos de sorbato de potássio $(0,1 \%)$, independentemente do teor de sacarose, e $\mathrm{b}^{*}$ foi maior para os teores máximos de sorbato de potássio e mínimo de sacarose.

A Tabela 5 apresenta os dados dos coeficientes de correlação entre as medições objetivas e subjetivas da cor. Estes dados mostraram maiores coeficientes entre a ordenação visual da luminosidade (OVL), vermelho (OVV) e amarelo (OVA) com o valor de $b^{*}$ (amarelo), ou seja, os julgadores ordenaram as amostras tendo como parâmetro principal o amarelo. Os coeficientes de correlação foram ligeiramente melhorados nas correlações múltiplas da ordenação pela intensidade de vermelho e valores objetivos de cor. Foram observados coeficientes de correlação entre o croma (C) e a ordenação de vermelho (OVV). 


\section{TABELA 5 - COEFICIENTES DE CORRELACÃO LINEAR E MÚLTIPLA ENTRE OS DADOS DAS MEDIÇÕES OBJETIVAS E SUBJETIVAS DA COR DAS POLPAS DE GOIABA ESTÁVEIS}

\begin{tabular}{l|c|c|c}
\hline \multirow{2}{*}{$\begin{array}{c}\text { MEDIÇÕES } \\
\text { OBJETIVAS }\end{array}$} & OVL & OVV & OVA \\
\cline { 2 - 4 } & 0,8613 & 0,9369 & $-0,8825$ \\
Luminosidade (L*) & 0,8614 & 0,8024 & $-0,8218$ \\
Vermelho (a*) & 0,9240 & 0,9601 & $-0,9263$ \\
Amarelo (b*) & 0,9125 & 0,9435 & 0,9074 \\
$\mathrm{~L}^{*} \mathrm{a}^{*}$ & 0,9359 & 0,9603 & 0,9289 \\
$\mathrm{~L}^{*} \mathrm{~b}$ & 0,9295 & 0,9643 & 0,9264 \\
$\mathrm{a}^{*} \mathrm{~b}^{*}$ & 0,9360 & 0,9656 & 0,9297 \\
$\mathrm{~L}^{*} \mathrm{a} \mathrm{b}^{*}$ & 0,9289 & 0,9401 & $-0,9198$ \\
Croma $(\mathrm{C})$ & &
\end{tabular}

OVL = Ordenação Visual de Luminosidade; OVV = Ordenação Visual do Vermelho e OVA = Ordenação Visual do Amarelo.

\section{CONCLUSÃO}

Com relação à cor da polpa de goiaba, a luminosidade diminuiu com o aumento do teor de sacarose, o teor de vermelho foi maior com a adição de $0,1 \%$ de sorbato de potássio e o teor de amarelo foi maior para $0,1 \%$ de sorbato de potássio e $0 \%$ de sacarose. Os tratamentos aplicados diminuiram a luminosidade, o teor de vermelho e amarelo das amostras com relação a polpa de goiaba inicial, com exceção do tratamento com $0 \%$ de sacarose, $0,75 \%$ de ácido cítrico e $0,10 \%$ de sorbato de potássio, que conferiu a amostra dados bastante próximos ao da polpa inicial.

\section{Abstract}

Seven samples of guava pulp stable to the storage at $25^{\circ} \mathrm{C}$, preserved with the use of the technology of combined methods and treated with sucrose, citric acid and potassium sorbate were evaluated for color variations in relation to the original sample (without treatment). Color measurement were taken by reflection in spectrophotometer. With the obtained data it was calculated the chrome and the total difference of the color in relation to the original sample. All the treatments were visual evaluated and ordered based on the luminosity degree, red and yellow by a superior color discrimination panel. All applied treatments decreased the luminosity of the samples, redness and yellowness compared to the original guava pulp, except to the treatment $1(0 \%$ sucrose, $0,75 \%$ citric acid and $0,10 \%$ potassium sorbate) that showed values very similar to the original pulp. 


\section{REFERÊNCIAS}

1 ALZAMORA, S. M.; GERSCHENSON, L. N.; CERRUTI, P.; ROJAS, A. M. Shelf-stable pineapple for long-term non-refrigerated storage. Lebensmittel-Wissenschaft-und-Technologie, Londres, v. 22, n. 5, p. 233-236, 1989 .

2 CHIRIFE, J.; FAVETO, G. J. Some physico-chemical basis of food preservation by combined methods. Food Research International, Oxford, v. 25, n. 5, p. 389-396, 1992.

3 CYTED. Programa Iberoamericano de Ciencia y Tecnologia para el Desarrolo. Aportaciones al desarrolo tecnologico de los alimentos de humedad intermedia preservados mediante metodos combinados. Puebla: Universidad de las Américas, 1991. 136 p. (CYTED-D. Informacion Tecnica, 4).

4 FERREIRA, V. L. P. Princípios e aplicações da colorimetria em alimentos. Campinas: ITAL, 1981. 85 p. (Instruções Técnicas, 19).

5 FERREIRA, V. L. P.; FRANCIS, J. F.; YOTSUYANAGI, K. Cor e carotenóides totais em suco de maracujá (Passiflora edulis, Sims.). Coletânea do ITAL, Campinas, v. 19, n. 1, p. 50-58, 1989.

6 FOX, M.; LONCIN, M. Investigations into the microbiological stability of water-rich foods processed by a combination of methods. Lebensmittel-Wissenschaft-und-Technologie, Londres, v. 15, n. 6, p. 321-325, 1982.

7 FRANCIS, J. F.; CLYDESDALE, F. M. Food colorimetry: theory and application. Westport: AVI, 1975. 447 p.

8 HOFFMANN, R.; VIEIRA, S. Análise de regressão: uma introdução à econometria. 2.ed. São Paulo: Hucitec, 1987. 379 p.

9 JAYARAMAN, K. S.; RAMANUJA, M. N.; BHATIA, B. S.; NATH, H. Some studies on the preparation of intermediate moisture guava. Journal of Food Science and Technology, Mysore, v. 11, n. 4, p. 162-165, 1974.

10 JAYARAMAN, K. S. Development of intermediate moisture tropical fruit and vegetable products-technological problems and prospects. In: SEOW, C.C.; TENG, T.T.; QUAH, C.H. Food preservation by moisture control. Londres: Elsevier Applied Science, 1988. p. 175198. 
11 LEISTNER, L. Principles and aplications of hurdle technology. In: GOULD, G.W. (ed.). New methods of food preservation. Londres: Chapman \& Hall, 1995. p. 1-22.

12 LÓPEZ-MALO, A.; PALOU, E.; WELTI, J.; CORTE, P.; ARGAIZ, A. Shelf-stable high moisture papaya minimally processed by combined methods. Food Research International, Oxford, v. 27, n. 6, p. 545553, 1994.

13 NEWEL, G. J.; MacFARLANE, S. D. Expanded tables for multiple comparison procedures in the analysis of ranked data. Journal of Food Science, Chicago, v. 52, n. 6, p. 1721-1725, 1987.

14 PADULA, M.; RODRIGUES-AMAYA, D. B.; MORAES, M. A. C. Comparison of the carotenoid composition and general properties of the processed juice of guava cultivar IAC-4 and commercial juices. Ciência e Tecnologia de Alimentos, Campinas, v. 3, n. 2, p. 109116, 1983.

15 SPIEGEL, M. R. Estatística. São Paulo: McGraw Hill, 1974. 580 p.

16 TORREZAN, R. Preservação de polpa de goiaba por métodos combinados. Campinas, 1996. 211 p. Dissertação (Mestrado), Faculdade de Engenharia de Alimentos, Universidade Estadual de Campinas.

17 WILBERG, V. C.; RODRIGUEZ-AMAYA, D. B. HPLC quantitation of major carotenoids of fresh and processed guava, mango and papaya. Lebensmittel-Wissenschaft-und-Technologie, Londres, v. 28, n. 5, p. 474-480, 1995. 